\title{
Modelling Drying Kinetics of Black Soldier Fly (Hermetia Illucens, L.) Larvae
}

\author{
Pascoal da Silva ${ }^{1,3}$, Nuno Ribeiro ${ }^{2}$, Maria Nazaré Coelho Pinheiro ${ }^{1,4^{*}}$, and Rui Costa ${ }^{2}$ \\ ${ }^{1}$ Instituto Politécnico de Coimbra, Instituto Superior de Engenharia de Coimbra, Rua Pedro Nunes, Quinta da Nora, 3030-199 Coimbra, \\ Portugal \\ ${ }^{2}$ CERNAS, Instituto Politécnico de Coimbra, Escola Superior Agrária, Bencanta, 3045-601, Coimbra, Portugal \\ ${ }^{3}$ Centro de Matemática da Universidade de Coimbra, Apartado 3008, EC Santa Cruz,3001 - 501 Coimbra, Portugal \\ ${ }^{4}$ Centro de Estudos de Fenómenos de Transporte, Faculdade de Engenharia da Universidade do Porto, Rua Dr. Roberto Frias, 4200-465 \\ Porto, Portugal
}

\begin{abstract}
Hermetia illucens (Black Soldier Fly) larvae (BSFL) have great potential as new protein source for either feed or food use due to the high content of protein and fat. BSFL may be used in different forms including dried with extended shelf life.

Mathematical models describing dehydration kinetics are useful to optimize BSFL drying conditions. A non-stationary 3D diffusion model, given by Fick's Law was used to describe the moisture depletion inside BSFL during drying. The numerical solution of PDE was obtained with COMSOL Multiphysics assuming simple boundary conditions.

Data were obtained in a drying experiment performed with fresh larvae uniformly laid on a metallic grid in a forced air oven at $80{ }^{\circ} \mathrm{C}$. Five randomly larvae were periodically selected and removed for moisture content determination until equilibrium moisture was reached.

A qualitative good agreement between predictions and data was observed corroborating that the main physical phenomena were considered in the mathematical description. A better quantitave description is expected with the improvement of the boundary condition at larvae surface in the model and the upgrading of the three-dimensional geometry used as domain for the numerical resolution.
\end{abstract}

\section{Introduction}

Due to continuing growth of the world population, that is expected to reach 9.3 billion of people by 2050 and the raising of living standards in newly industrialized countries with the consequent increase of food consumption per capita, the demand for food will continue to grow to unsustainable levels [1]. Consequently, the demand for the most common sources of protein, soybean meal (food, feed and biodiesel production) and fishmeal (essentially for feed) had been increasing with known consequences for natural resources and the environmental system. Thus, the search for environmentally sustainable and financially viable alternative sources of protein is one of the most crucial factors in the development of intensive exploration in the future. Alongside with algae and pulses, insects are foreseen as one of the best options to contribute to achieve the required levels of sustainability in food and feed production.

Insect rearing as a novel source of protein both to human food and animal feed has been gaining followers and several production projects at an industrial scale already exist. Insects can grow and reproduce quickly and easily, have high feed conversion efficiency while contribute to food and feed security [2].

Though insects are the largest groups of living organisms and had always been used as food and feed all over the world [3], only a few insect species have been commercially mass produced for a protein source, either in an industrial or a "cottage" setup.

One of those insects is the Hermetia illucens (Black Soldier Fly, BSF) due to its versatility regarding the substrates it can feed on, the absence of known zoonosis, its low potential to become an invasive species (given its biological requirements for high temperatures and high relative humidity to reproduce successfully) and its range of uses and final products. BSF larvae (BSFL) have been used for feed (swine, poultry or fish) [4] and for biodiesel production [5].

BSFL can be fed fresh and directly to some animals but, at industrial scale, drying is one of the best methods to increase shelf-life, reducing water activity, and to reduce volume and weight for storage products [6].

To our knowledge, no study on drying kinetics of BSF has been published. Thus, a systematic approach for understanding the drying dynamics of the BSFL is of the utmost importance to optimize the storage and packaging larvae processes. The aim of this study is, therefore,

\footnotetext{
* Corresponding author: mnazare@isec.pt
} 
characterize and modelling the drying kinetics of the BSFL.

\section{Materials and methods}

\subsection{BSFL characterization}

The morphology and modifications during lifecycle of BSFL were object of study by different research teams. Oliveira et al. [7] used SEM (Scanning Electron Microscopy) to analyze and document the anatomy of the larval stage of BSF. Larvae have an elongated shape with the body divided in three main sectors: head; thorax (with three segments) and abdomen (with eight segments).

Live BSFL were supplied from the colony of ESAC (Escola Superior Agrária de Coimbra) located in Bencanta, Coimbra (Portugal). After collection the larvae were rinsed and frozen at $-80{ }^{\circ} \mathrm{C}$ until the experiments. Defrozen larvae at ambient temperature hereafter are considered as fresh.

In order to characterize the material to be dried, a significant number of fresh larvae was measured individually. Using a digital caliper the thickness of larvae was obtained and measurements of length and diameter were performed through an image using ImageJ (U.S. National Institutes of Health, Bethesda, Maryland, USA), after calibration with a scale. Diameter was measured in three different locations: in the first thoracic segment (near the head); in the last abdominal segment and in the middle of the dorsal side. The mean values obtained from individual measurements of a sample of 87 larvae are presented in Table 1. The standard deviation (S.D.), the $95 \%$ confidence interval (C.I.) and the median for the larvae population are also indicated in the table.

Table 1. Mean values for fresh larvae dimensions obtained from a population of 87 units.

\begin{tabular}{lccccc}
\hline & $\begin{array}{c}\text { Thickness } \\
(\mathrm{mm})\end{array}$ & $\begin{array}{c}\text { Length } \\
(\mathrm{mm})\end{array}$ & \multicolumn{3}{c}{$\begin{array}{c}\text { Diameter at dorsal side } \\
(\mathrm{mm})\end{array}$} \\
\hline & & & thorax & middle & abdomen \\
\hline mean & 3.4 & 16.7 & 3.0 & 4.7 & 3.5 \\
S.D. & 0.3 & 1.8 & 0.5 & 0.6 & 0.6 \\
95\% C.I. & 0.1 & 0.4 & 0.1 & 0.1 & 0.1 \\
median & 3.5 & 16.6 & 3.0 & 4.6 & 3.5 \\
\hline
\end{tabular}

The mean value of the dimensions measured in the larvae sample was used to characterize the geometry of the humid material to be dried. It should be emphasized that the larvae ventral side is almost flat and the dorsal side is rounded.

\subsection{Drying kinetics experiment}

After defrosting at temperature room $\left(\approx 22{ }^{\circ} \mathrm{C}\right)$, the larvae were weighed and uniformly distributed with gaps between them in a rack and placed in the forced air oven (model STF-F 52-Lt; Falc co., Treviglio, Italy) at $80{ }^{\circ} \mathrm{C}$. During the process, ten different sampling times were chosen based on pre-experiment trials to evaluate the time needed to reach the equilibrium condition. At each time, five different samples of larvae were randomly selected and weighed for moisture content determination. Then the fifty larvae were placed again in the oven at $105^{\circ} \mathrm{C}$ until a constant weight was obtained and their moisture content determined.

The moisture content $(\mathrm{X})$ evolution within larvae during drying was obtained in a dry basis as,

$$
X=\frac{m-m_{S}}{m_{S}}
$$

where $m$ is the weight of the larvae removed from the oven at instant $t$ and $m_{s}$ is the final weight of the larvae dried at $105^{\circ} \mathrm{C}$.

The dimensionless solid moisture content, the moisture ratio $(M R)$, is defined as,

$$
M R=\frac{X-X_{\mathrm{e}}}{X_{\mathrm{i}}-X_{\mathrm{e}}}
$$

and is the ratio between the free moisture $\left(X-X_{\mathrm{e}}\right)$ inside the larvae at instant $t$ and its initial free moisture $\left(X_{\mathrm{i}}-X_{\mathrm{e}}\right) ; X_{\mathrm{e}}$ and $X_{\mathrm{i}}$ are the larvae moisture content when the equilibrium condition was reached and at the initial state, respectively.

\subsection{Drying kinetics modelling}

Simulate numerically drying kinetics can be a complex task since heat and moisture transfer occur simultaneously throughout the solid, followed by moisture evaporation at the solid surface. Moreover, during drying the solid can be forced to experience texture modifications with dimensions retraction. However, introducing some assumptions results in simple models with a reasonable description of the drying operation.

Assuming moisture diffusion as the only mechanism of water transport throughout the solid, the diffusion equation can be written as

$$
\frac{\partial X}{\partial t}+\nabla \bullet\left(-D_{e f f} \nabla X\right)=0 \text { in } \Omega,
$$

where $\Omega$ stands for the domain that represents the larvae and the medium where diffusion occurs. Considering a homogeneous and isotropic domain, the following equation can be considered

$$
\frac{\partial X}{\partial t}-D_{e f f} \nabla^{2} X=0 \text { in } \Omega
$$

For the sake of simplicity, the larvae dimensions and the moisture effective diffusivity (Deff) were considered constant during drying and independent of the moisture content.

Initially, the moisture distribution inside the larvae was assumed uniform 


$$
X(\Omega, t=0)=X_{i}
$$

and this equality was used as the initial condition in the diffusion equation, together with the following boundary conditions

$$
X(t)=X_{e} \text { in } \partial \Omega_{1}
$$

where $\partial \boldsymbol{\Omega}_{1}$ represents the external surface of the domain $\Omega$.

The mean value of moisture inside larvae for a given instant $t$, is determined by

$$
\bar{X}(t)=\frac{1}{V} \iiint_{V} X(\Omega, t) d V
$$

with $V$ representing the larvae volume.

\subsection{Effective moisture diffusivity prediction}

The estimation method used to obtain the effective moisture diffusivity was based on Fick's law for diffusion using drying data. The solution of the Fick's second law, for one direction diffusion, is well known when some simplifications are assumed: $i$ ) constant diffusivity; ii) initial moisture distribution uniform and iii) moisture at the humid material surface equal to the equilibrium moisture content. Crank [8] presents solutions for infinite slabs, infinite cylinders and spheres. Time integration of the analytical solution for an infinite slab [8], gives the moisture ratio (MR) inside larvae as a function of time $(\mathrm{t})$,

$$
M R=\frac{8}{\pi^{2}} \sum_{n=0}^{\infty} \frac{1}{(2 n+1)^{2}} \exp \left[-\frac{(2 n+1)^{2} \pi^{2} D_{\text {eff }} t}{4 L^{2}}\right]
$$

where $L$ stands in the present situation for the half of medium thickness because symmetric drying conditions exists in both sides of the larvae.

The infinite series in the solution converges rapidly increasing the time and keeping the first term results in small errors. Therefore, a useful approximation can be written as:

$$
M R=\frac{8}{\pi^{2}} \exp \left(-\frac{\pi^{2} D_{e f f} t}{4 L^{2}}\right)
$$

A linear correlation is obtained between $\ln M R$ and $t$ with slope $-\pi^{2} D_{\text {eff }} / 4 L^{2}$ and the value of $D_{\text {eff }}$ can be calculated from the larvae drying experiment.

The representation obtained from data collected during the drying experiment conducted at $80{ }^{\circ} \mathrm{C}$ is presented in Figure 1. A good linear correlation can be observed

with a coefficient of determination equal to 0.9520 and slope $5.0312 \times 10^{-4} \mathrm{~s}^{-1}$. A value of $D_{\text {eff }}$ equal to $1.0703 \times 10^{-10} \mathrm{~m}^{2} / \mathrm{s}$, at $80{ }^{\circ} \mathrm{C}$, was calculated and will be used as input during numerical resolution of the simple model proposed. It should be noted that previously was evaluated the drying rate curve behaviour and no evidence of a period of constant drying rate was observed. Hence, a falling drying rate period was considered during entire drying process, where the Fick's second law of diffusion can be applied, and all data collected during experiment was used to estimate $D_{\text {eff. }}$.

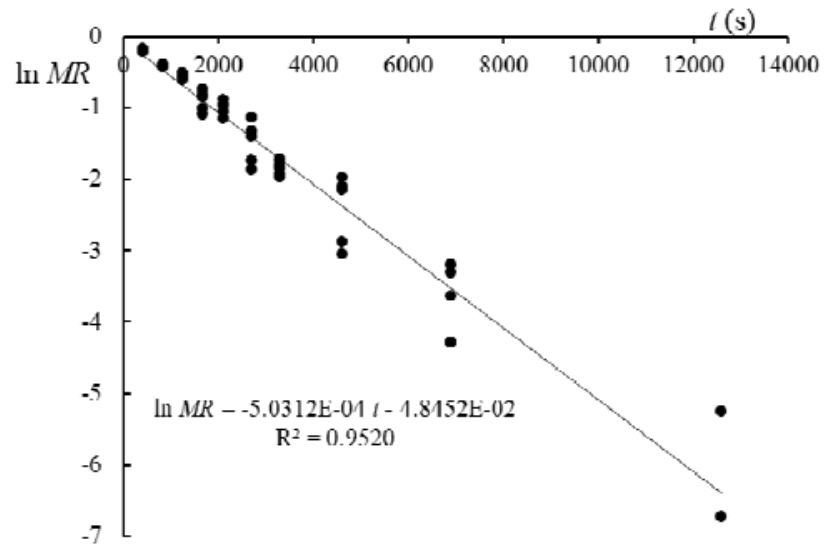

Fig. 1. $\ln M R$ versus $t$ for effective diffusion coefficient estimation from BSFL drying kinetics data, at $80^{\circ} \mathrm{C}$.

\subsection{Numerical resolution}

The mean dimensions of the fresh larvae population (see Table 1) were used to generate the three-dimensional domain where the simulations were obtained.

The three-dimensional domain representing half of a larvae was generated using a revolve procedure to a work plane with the surface created with the larvae dimensions measured, shown in Figure 2a). The mathematical model presented (Equations 4 to 6 ) is solved considering a symmetrical boundary condition at $\partial \Omega_{2}$ (the plane of symmetry shown in Figure 2b)), with COMSOL Multiphysics, using a piecewise finite element method. A mesh automatically generated with 265744 tetrahedral elements is used and it is represented in Figure 3a). In Figure 3b) a larvae photography is shown for comparison with the domain generated.

To integrate in time (Equation 4), adaptive Backward Differentiation Formulae, with orders between 1 and 2 and adaptive time step are used.

\section{Results and discussion}

In order to simulate the diffusional problem associated to larvae drying is essential to recreate the medium geometry where moisture diffusion occurs based on a dimensional characterization of a larvae population (indicated at Table I) and use realistic inputs for PDE numerical resolution.

The numerical simulations were obtained with $X_{\mathrm{i}}=$ $2.5269 \mathrm{~kg}_{\text {water }} / \mathrm{kg}_{\text {dry solid }}$ and $X_{\mathrm{e}}=0.0262 \mathrm{~kg}_{\text {water }} / \mathrm{kg}_{\text {dry solid, }}$ the mean values calculated for the moisture content within larvae at the beginning of drying process and when equilibrium conditions were reached, respectively. 
The value of $D_{\text {eff }}$ estimated according the methodology described previously is also an important input parameter.

a)

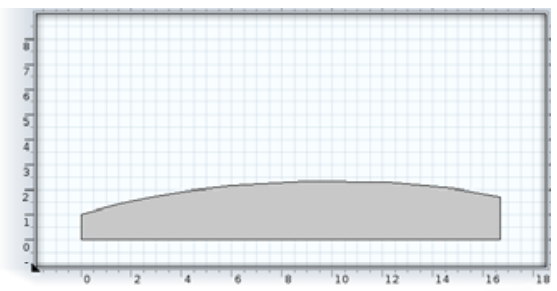

b)

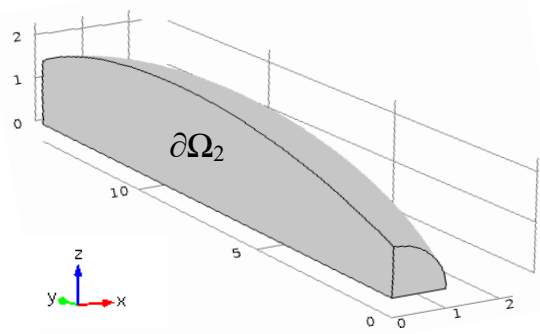

Fig. 2. Surface created in the work plane with the mean larvae dimensions a) and 3D geometry created revolving the surface b). a)

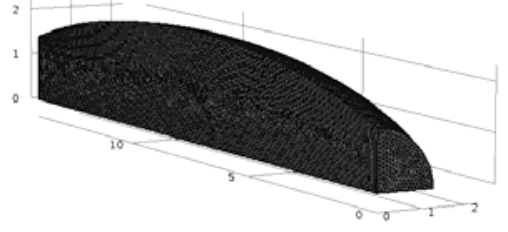

b)

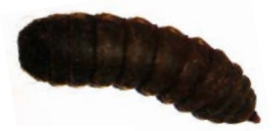

Fig. 3. Mesh built for the 3D geometry created a) and photography of a fresh BSFL b).

In Figure 4 the moisture distribution is exhibited for three different instants ( $2 \mathrm{~min}, 1 \mathrm{~h}$ and $3 \mathrm{~h}$ ) during BSFL drying at $80{ }^{\circ} \mathrm{C}$. After 2 min of drying, the moisture in the inner region within larvae is almost uniform and equal to $X_{\mathrm{i}}$. Only over the periphery, in contact with the drying agent (hot air), a moisture depletion is observed due to the moisture gradients developed because $X_{\mathrm{e}}$ was imposed at the outer larvae surface (see Equation 6). As expected, the moisture front recedes inside the larvae as the drying progresses. After $3 \mathrm{~h}$ only in the most inner region, the moisture gradients promoting diffusion are significant.

The average moisture content inside the larvae at instant $t$ determined by spatial integration over all domain $\Omega$ (see Equation 7) is useful for comparison with data obtained in the drying experiment. The time evolution of $\bar{X}$ during drying predicted from simulations (full red line) is represented in Figure 5 together with data (symbols). From the moisture content decrease presented within larvae, as referred before, is not evident a constant drying rate period. In fact, a clear linear decrease of $\bar{X}$ with time is not observed after the apparently short unsteady state period in which the larvae, initially at ambient temperature, adjusted its temperature to drying conditions. A nearly entire falling drying rate period exists until the moisture content in the larvae remains approximately constant and the equilibrium with the air humidity prevailing in the airdrying atmosphere is achieved.
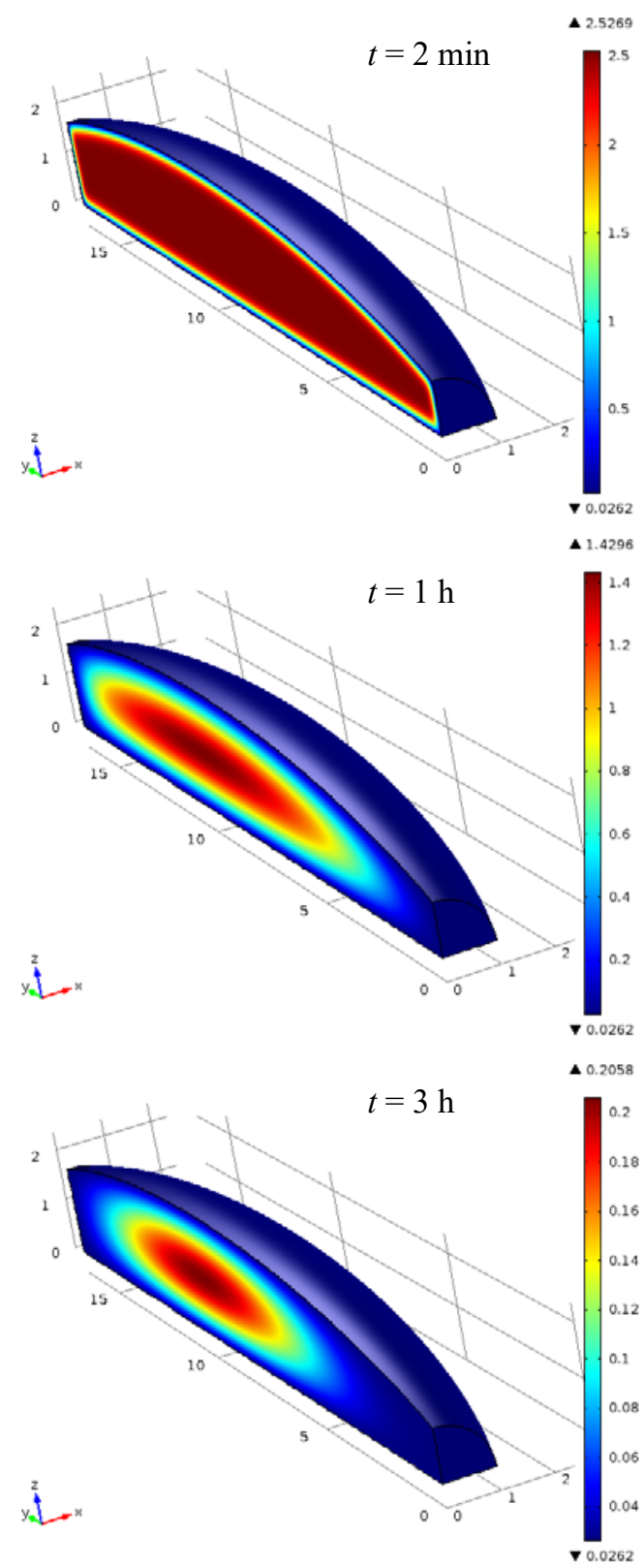

Fig. 4. Moisture distributions in the larvae during drying obtained from simulations. The moisture content scale is different for the three plots presented.

From Figure 5, simulations and experiments are qualitatively in accordance. Thus, it seems that the simple model considered includes the principal phenomena occurring during the falling drying rate period. However, the predicted removal of moisture from larvae is overestimated at the beginning of drying process and underestimated thereafter. 
The quantitative discrepancy shown in Figure 5 between predictions and data is not very significant attending the assumptions made during model development. A future improvement of the boundary condition imposed at larvae surface exposed to air drying conditions and an upgrading of the medium geometry recreated must be considered to increase the model performance. Moreover, the retraction in the larvae dimensions and the dependence of $D_{\text {eff }}$ with the larvae moisture content during drying should also be taken into consideration to improve predictions.

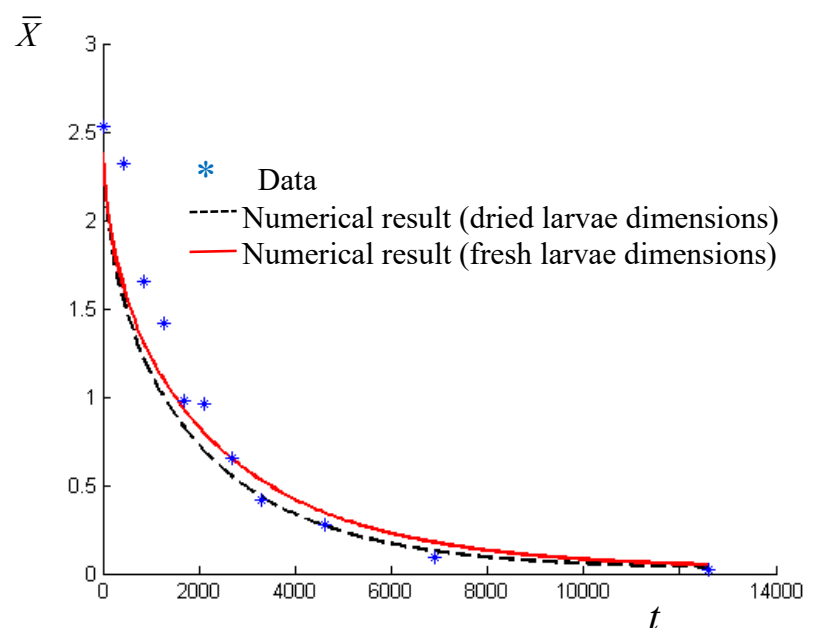

Fig. 5. Average moisture content as a function of time for BSFL drying at $80^{\circ} \mathrm{C}$. Full red line (fresh larvae dimensions) and dashed black line (dried larvae dimensions) are predictions and symbols correspond to data.

In order to explore the effect of larvae shrinkage a simple exercise was implemented. A simulation were performed using the mean dried larvae dimensions to generate the three-dimensional domain where the numerical results were obtained. With this purpose, larvae dimensional characterization were made also after being dried using a measuring methodology similar to the one described for the fresh larvae. As expected, all mean values obtained from measurements were smaller than dimensions indicated in Table 1 for fresh larvae. The mean length obtained for dried larvae was $(12.4 \pm$ 1.4) $\mathrm{mm}$, representing a decrease of $\approx 26 \%$. The diameter at thorax, middle and abdomen of larvae dorsal side, was reduced to $(1.9 \pm 0.4) \mathrm{mm},(4.4 \pm 0.7) \mathrm{mm}$ and $(2.4 \pm 0.5 \mathrm{~mm})$, respectively. Retraction in diameter during drying process is significant mainly in the larvae extremes.

The plot for time evolution of $\bar{X}$ during drying predicted with the larvae dried dimensions to generate the three-dimensional domain is represented also in Figure 5 (dashed black line). The effect of larvae dimensions is significant in the predictions obtained after $1000 \mathrm{~s}$ of drying. After $3000 \mathrm{~s}$ there is a very good agreement between results simulated and data, indicating that when moisture gradients within larvae decrease using realistic medium dimensions to recreate the geometry is essential. Moreover, the simple boundary condition defined imposing a fixed moisture content at larvae surface equal to the equilibrium content (a Dirichlet boundary condition) is approaching for high drying times.

It is our expectation that improving the boundary conditions at larvae surface with a flux condition (a Neumann boundary condition) in the model and recreating a medium geometry with variable dimensions to account for larvae shrinkage during drying for PDE numerical resolution will increase the model performance significantly.

\section{Conclusions}

A simple model to describe the unsteady state diffusion of moisture through BSFL during drying was implemented and numerical solutions were obtained using COMSOL Multiphysics. Simultaneously, an experiment was carried out to evaluate the BSFL drying kinetics at $80^{\circ} \mathrm{C}$.

From the evolution with time of the BSFL moisture content determined from data, a unique falling drying rate period seems to exist, indicating that diffusion is the main mass transfer mechanism. The prediction of the effective moisture diffusion coefficient was made using drying kinetics data and a value equal to $1.0703 \times 10-10$ $\mathrm{m} 2 / \mathrm{s}$, at $80^{\circ} \mathrm{C}$, was obtained.

The simple mathematical approach presented was applied successfully to the drying process of BSFL, however it deserves improvements to obtain better agreement between predictions and data.

Significant BSFL shrinkage was observed during drying experiment and a simple exercise performed with dried larvae dimensions revealed that this phenomenon should be implemented in the simulations. Replace the Dirichlet boundary condition defined at BSFL surface by a Neumann boundary condition will be an improvement to implement in the model in order to enhance the simulations agreement with data.

The work presented represents an advance in the knowledge of BSFL as potential source of protein in the future for human nutrition. To our knowledge, this is the first study to be published in the topic of BSFL drying kinetics.

Acknowledgments: The authors would like to thank Pablo Goenaga for his assistance in the drying experiment.

\section{References}

1. FAO, FAO's Director-General on How to Feed the World in 2050, Popul. Dev. Rev. 35, 4, 837-839 (2009).

2. A. van Huis, J. van Itterbeeck, H. Klunder, E. Mertens, A. Halloran, G. Muir, P. Vantomme, Edible Insects Future Prospects for Food and Feed Security, FAO, Rome (2013).

3. G.R. DeFoliart, An Overview of the Role of Edible Insects in Preserving Biodiversity, Ecol. Food Nutr. 36, 2-4, 109-132 (1997). 
4. K.B., Barragan-Fonseca, M. Dicke, J.J.A. van Loon, Nutritional Value of the Black Soldier Fly (Hermetia Illucens L.) and Its Suitability as Animal Feed - a Review, J. Insects as Food Feed 3, 2, 105120 (2017).

5. F. Manzano-Agugliaro, M.J.J. Sanchez-Muros, F. G.G. Barroso, A. Martínez-Sánchez, S. Rojo, C. Pérez-Bañón, Insects for Biodiesel Production, Renew. Sustain. Energy Rev. 16, 6, 3744-3753 (2012).

6. Y. Kumar, S. Tiwari, S.A. Belorkar, Drying: An Excellent Method for Food Preservation, Int. J. Eng. Stud. Tech. Approach 01, 8 (2015).

7. F. Oliveira, K. Doelle, R. List, J.R. O'Reilly, Assessment of Diptera: Stratiomyidae, genus Hermetia illucens (L., 1758) using electron microscopy, J. of Entomology and Zoology Studies 3, 5, 147-152 (2015).

8. J. Crank, The Mathematics of Diffusion (Oxford University Press: New York ,1975). 